\title{
Spectroscopic approaches to the measurement of food quality
}

\author{
P. S. Belton \\ Institute of Food Research, Norwich Laboratory, Norwich Research Park, \\ Colney, Norwich NR4 7UA, UK.

\begin{abstract}
:
The richness of the electromagnetic spectrum is well matched by the range of problems that are inherent in food science. Foods are typically heterogeneous and contain a very wide range of materials in a variety of physical states. Different states often coexist in a non-equilibrium condition which may be perturbed by invasive techniques. Almost all of spectroscopy can, and does, play a role in food science. For the measurement of quality related parameters however two techniques, NMR and vibrational spectroscopy, have proved to offer an exceptionally wide range of uses. It is with these that this report is
\end{abstract} \\ mainly concerned.
}

\section{INTRODUCTION}

The electromagnetic spectrum offers about 17 orders of magnitude in energy. At the very high frequency end gamma rays and hard X-rays excite nuclear transitions. Such transitions typically carry very little information about chemical environments and can only give information about nuclear states and provide methods of elemental analysis. An exception to this is Moessbauer Spectroscopy in which sensitivity to the chemical environment is obtained by the observation of very small frequency shifts relative to the frequency of the exciting radiation.

At lower frequencies soft $\mathrm{x}$-rays and high frequency ultraviolet radiation excite transitions among core electrons. These too are relatively insensitive to the chemical environment and offer mainly information about elemental content. With lower energy ultraviolet radiation the first real sensitivity to chemical environment appears. This is because the lower energy UV excites transitions among valence electrons which are directly involved in chemical bonding. Typically however UV transitions are broad and the degree of chemical sensitivity is limited. In the visible region lower energy valence electron transitions are excited but chemical sensitivity is not significantly greater than in the UV region.

In the near infrared (NIR) region molecular, rather than electronic, transitions are excited by way of the overtones and combinations of chemical bond vibrations. This spectral region has been exploited very heavily by food scientists. Water absorbtions are relatively weak and diffuse reflectance techniques work very well. However the vibrational modes involved are limited to those bonds which contain hydrogen atoms.

The mid infrared (MIR) region has a very high level of sensitivity to chemical environment as it is the region in which most of the fundamentals of molecular vibrations occur. It is very rich in information on both molecular composition and the physical state of the absorbing molecules. The far infrared and microwave regions carry information about very low frequency vibrations and rotational motion. The microwave region is also the usual region in which electron paramagnetic resonance (EPR) is observed. EPR is a magnetic resonance spectroscopy in which transitions between magnetic states of electrons are excited. Typically these occur in transition metals or in synthetic or natural free radicals. 
The radio frequency region is where nuclear magnetic resonance (NMR) spectroscopy is normally carried out, although with the advent of higher and higher field spectrometers frequencies are now beginning to approach the microwave region. NMR is a nuclear spectroscopy. It owes its exquisite chemical and physical sensitivity to the fact that the width of the nuclear transitions observed are very narrow compared to the exciting wavelength, an analogy to Moessbauer spectroscopy.

Clearly most, if not all, of the electromagnetic spectrum has something to offer the food scientist. Applications of spectroscopy to food science (1) and the spectroscopic context of NMR (2) are discussed in more detail elsewhere. In this report only two types of spectroscopy are considered in detail. These are mid infrared and NMR. These have been chosen because of the wealth of information that they provide on all aspects of food quality.

The problem for the spectroscopist in the elucidation of food quality is that food originates from a very wide variety of plant and animal sources. These may be eaten fresh but much more frequently are processed or combined in some way. The processing may take the simple form of cooking, but more usually there are mixing or other mechanical treatment stages designed to change the physical and or chemical state of the food. Sometimes fermentation using fungi or bacteria is involved and this process may be combined with the others in order to produce the consumed product. Bread is a very common example in which mechanical, fermentative and cooking stages are involved. Even after the complexities of the preparation stage there may be spoilage of the food during storage due to chemical changes or due to biological attack. The delicate balance of physical, chemical and biological forces is critical to the quality of the final product and may be easily changed by invasive methods. The challenge to spectroscopy and the spectroscopist is therefore a daunting one.

\section{NUCLEAR MAGNETIC RESONANCE}

The great advantage of NMR in such complex systems such as food is that the spectroscopic method naturally filters the information that is observed by the spectrometer. At the most basic level, signals from only one nucleus are observed at a time. This arises because the magnetogyric ratios of the nuclei of different isotopes ensure that the signals from different isotopes, and hence elements, are well separated. Typically therefore, provided the nuclei chosen are not carbon or hydrogen, relatively simple spectra may be observed.

A good example of the use of elemental specificity to simplify observation is the Phosphorus NMR spectrum of milk and milk products $(3,4)$. The use of ${ }^{31} \mathrm{P}$ NMR enables the selection of only the signals originating from phosphorus bearing species. In this way inorganic phosphate species as well as phosphate esters and phosphoserine could easily be observed in a relatively simple spectrum.

Because the sensitivity of NMR is isotopic rather than elemental, isotopic substitution can be used to great effect. Thus it is possible to enhance signals from isotopes with low natural abundance or replace a resonant nucleus with a non-resonant one. This is very useful in aqueous systems where it is often possible to replace ${ }^{1} \mathrm{H}_{2} \mathrm{O}$ with ${ }^{2} \mathrm{H}_{2} \mathrm{O}$. In this way the proton signal from the water can be eliminated and the less intense resonances from, for example, biopolymers (5) may be seen. An alternative method of using the isotopic selectivity of NMR is to introduce a nucleus that does not normally occur in the system as a probe for local properties of interest. This is rather similar in approach to the use of spin probes in EPR. A probe nucleus that has been used to very good effect in synthetic polymers is Xenon (6). It is very sensitive to cavities with dimensions of molecular scale and larger and, of course, is inert in almost all circumstances. It is not always necessary to use exotic species. ${ }^{23} \mathrm{Na}$ has been used as probe for local dynamical conditions in non-freezing water around proteins at sub zero temperatures (7).

NMR is not limited to the filtration of information by isotope selection. It is normal to observe signals from nuclei in only a limited range of motional states. Typically high resolution solution state spectrometers can only observe signals from highly mobile molecules. In the solids linewidths are usually too wide to be observed except by the special techniques of solid state NMR. It is possible 
to obtain high resolution spectra of solids by the use of cross polarisation magic angle spinning methods (8). In this experiment only those nuclei with long motional correlation times are selected for observation. In general the experiment involves the transfer of polarisation from a nucleus with a high magnetogyric ratio, such as hydrogen, to one with a low ratio such as carbon. By subtle alteration of the details of the experiment nuclei in slightly differing motional states may be observed. In the case of wheat gluten it has been possible to observe the protein separately from the lipid fraction in the dry state (9) and thus to demonstrate that the lipid is more mobile than the protein and that it may act as plasticiser. The ability to analyse both chemical and motional states is of great value when trying to understand the functionality of complex biochemical systems.

Whilst the ability to eliminate unwanted information is valuable, there are cases where large amounts of information about the chemical composition of a system is very useful. The use of high field NMR spectrometers to obtain proton spectra of complex biological fluids is beginning to open the way to a very fast and complete analytical method for the liquid component of foods and, in particular, for drinks such as fruit juices and wine (10).

\section{MID INFRARED SPECTROSCOPY}

Mid infrared spectroscopy arises from the transitions associated with the vibrations of chemical bonds. Not all the possible vibrations of the system are observed since the selection rules for infra red absorption only allow transitions involving a change of the dipole moment to be observed. In practice this means that symmetric vibrations of bonds which have a centre of symmetry are either not observed or are weak. Typically, signals from completely symmetric molecules such as $\mathrm{O}_{2}$ or $\mathrm{N}_{2}$ are not observed at all. Because of the relatively high photon energy (compared to NMR) it is not generally possible to generate the conditions of coherence spectroscopy (2). Thus in essence, only one type of experiment is possible.

The use of Fourier transform methods combined with improved sampling techniques $(11,12)$ has enabled the spectra to be obtained from a wide range of food materials which are typically very opaque and unamenable to conventional sampling techniques. The applications are now very wide ranging. They fall into two main classes: analytical methods and conformational studies. Analytical methods may be limited to the simple application of Beer's law plots (11) or, as is increasingly the case, may make use of the sophisticated methods of multivariate statistics (13). When combined with multivariate methods the true power of infrared as an analytical technique becomes apparent since then the whole spectrum may be used so that subtle spectral differences can be exploited. In this way it has been possible to verify the origins of plant material for the purposes of authentication of foodstuffs (13). The use of the conformational sensitivity of infrared is well known in applications to protein secondary structure but it may also be applied to very good effect in the study of polysaccharide conformation. For example, it has proved possible to study the changes in starch conformation during the process of retrogradation (14) and to examine the way in which the conformation of the polymers of plant cell walls change during their sequential extraction (12).

\section{CONCLUSIONS}

Both NMR and infrared spectroscopy are of great value to food science and to the measurement of food quality, however it is defined. They may be used as analytical techniques or as subtle probes of the conformational and dynamical states of complex systems. As yet there has been relatively little in the food literature which has attempted to combine the two methods but it is likely that in combination they will prove to be highly complementary.

\section{ACKNOWLEDGMENT}

The author would like to thank the UK BBSRC for support. 


\section{REFERENCES}

1. R.H. Wilson, Spectroscopic Techniques for Food Analysis, VCH, New York (1994).

2. P.S. Belton, Ann. Reps. NMR Spectrosc. 31, 1, (1995).

3. P.S. Belton, R.L. Lyster and C. Richards, J Dairy Res.. 52, 47, (1985).

4. $\quad$ P.S. Belton and R.L. Lyster, J Dairy Res. 58, 443, (1991).

5. P.S. Belton, A.M. Gil and A.S. Tatham, J. Chem. Soc. Faraday Trans. 90, 1099, (1994).

6. D. Raftery and B.F. Chemlka, p111, NMR 30 Solid State NMR I (P. Diehl, E. Fluck, H. Gunther, R. Kosfeld and J. Seelig eds), Springer-Verlag, Berlin, (1994).

7. P.S. Belton and S. Kitchin, J. Chem. Soc. Faraday Trans. 91, 1459, (1994).

8. A-R. Grimmer and B. Bluemich, p1, NMR 30 Solid State NMR I (P. Diehl, E. Fluck, H. Gunther, R. Kosfeld and J. Seelig eds), Springer-Verlag, Berlin, (1994).

9. P.S. Belton, S.L. Duce and A. S. Tatham, Int. J. Biol. Macromol. 9, 357, (1987).

10. M. Spraul and M. Hofmann, P77, Magnetic Resonance in Food Science (P.S. Belton, I. Delgadillo, A.M. Gil and G.A. Webb eds), Royal Society of Chemistry, Cambridge (1995).

11. P.S. Belton A.M. Saffa and R.H. Wilson. Analyst, 112, 1117, (1987).

12. M. McCann, M. Hammouri, R.H. Wilson, P.S. Belton and K. Roberts, Plant Physiol. 100, 1940, (1992).

13. E.K. Kemsley, P.S. Belton, M. McCann, S. Ttofis, R.H. Wilson and I. Delgadillo, Food Control, 5, 241, (1994).

14. R.H. Wilson and P.S. Belton, Carbohydrate Res., 180, 339, (1988). 